\title{
Multiple massive domestication and recent amplification of Kolobok superfamily transposons in the clawed frog Xenopus
}

\author{
Akira Hikosaka ${ }^{*}$ (i) and Seigo Konishi
}

\begin{abstract}
Background: DNA transposons are generally destroyed by mutations and have short lifespans in hosts, as they are neutral or harmful to the host and therefore not conserved by natural selection. The clawed frog Xenopus harbors many DNA transposons and certain families, such as T2-MITE, have extremely long lives. These have ancient origins, but have shown recent transposition activity. In addition, certain transposase genes may have been "domesticated" by Xenopus and conserved over long time periods by natural selection. The aim of this study was to elucidate the evolutionary interactions between the host and the long-lived DNA transposon family it contains. Here, we investigated the molecular evolution of the Kolobok DNA transposon superfamily. Kolobok is thought to contribute to T2-MITE transposition.

Results: In the diploid western clawed frog Xenopus tropicalis and the allotetraploid African clawed frog Xenopus laevis, we searched for transposase genes homologous to those in the Kolobok superfamily. To determine the amplification and domestication of these genes, we used molecular phylogenetics and analyses of copy numbers, conserved motifs, orthologous gene synteny, and coding sequence divergence between the orthologs of $X$. laevis and $X$. tropicalis, or between those of two distant $X$. tropicalis lineages. Among $38 X$. tropicalis and $24 X$. laevis prospective transposase genes, 10 or more in $X$. tropicalis and 14 or more in $X$. laevis were apparently domesticated. These genes may have undergone multiple independent domestications from before the divergence of $X$. laevis and $X$. tropicalis. In contrast, certain other transposases may have retained catalytic activity required for transposition and could therefore have been recently amplified.
\end{abstract}

Conclusion: Multiple domestication of certain transposases and prolonged conservation of the catalytic activity in others suggest that Kolobok superfamily transposons were involved in complex, mutually beneficial relationships with their Xenopus hosts. Some transposases may serve to activate long-lived T2-MITE subfamilies.

Keywords: Xenopus laevis, Xenopus tropicalis, DNA transposon, Kolobok, T2-MITE, Transposase, Domestication, Molecular evolution

\section{Background}

Transposable elements (TEs) are endosymbiotic or parasitic genetic elements in the host genome. These elements usually replicate at their loci in the same manner as host genes. Nevertheless, they occasionally transpose to other loci and, in some cases, amplify themselves in the host genome. This non-Mendelian inheritance creates a conflict between TEs and their hosts.

* Correspondence: akirahs@hiroshima-u.ac.jp

Graduate School of Integrated Arts and Sciences, Hiroshima University,

Kagamiyama 1-7-1, Higashi-Hiroshima, Hiroshima, Japan
Transposable elements promote their amplification rates to increase their "fitness" in the host. In effect, their transposition and amplification are generally neutral, but occasionally detrimental, to the host as they increase mutations, such as gene disruption and ectopic recombination. In general, then, TE copies are not conserved. They are removed by purifying selection, and they lose their structure and function as they accumulate mutations.

TEs are classified into two major classes according to their transposition mechanisms: class I (RNA transposons or retrotransposons) and class II (DNA

(c) The Author(s). 2018 Open Access This article is distributed under the terms of the Creative Commons Attribution 4.0 International License (http://creativecommons.org/licenses/by/4.0/), which permits unrestricted use, distribution, and 
transposons) [1, 2]. An autonomous DNA transposon encodes its own transposase to catalyze its transposition between terminal inverted repeats (TIRs) including the transposase recognition site. In contrast, a nonautonomous DNA transposon has lost its own transposase gene owing to mutation(s). Its transposition depends on transposases provided by autonomous transposons coexisting in the genome. Autonomous transposons statistically lose their activity over time without purifying selection and become nonautonomous or nontransposable. Over time, the host genome will accumulate many nonautonomous copies and nontransposable "fossils" and retain relatively few autonomous copies. The TE family must keep producing autonomous copies to persist in the host. However, the transposase protein catalyzes the transposition of many nonautonomous copies and few autonomous copies, without distinguishing between them. Therefore, the probability that autonomous copies are transposed and amplified gradually decreases as the ratio of nonautonomous copies increases in the genome. Most DNA transposon families eventually become extinct in a host genome. This process is known as "vertical inactivation" [3, 4].

An alternative strategy for TE survival in a host is "molecular domestication." If a TE-derived gene, such as a transposase, has mutated and become beneficial to the host, it can be conserved by natural selection $[5,6]$. Domesticated genes typically lose their original nature (that is, the ability to catalyze transposition/amplification) and behave like host genes. Over time, domesticated genes diverge from their copies produced before domestication. As a result, they become and remain nonrepetitive (single-copy) genes. Domesticating a parasitic element is an important evolutionary innovation for hosts. Many TE-derived proteins have been domesticated [7, 8]. In the clawed frog Xenopus, we found that two subfamilies of the TxpB family belonging to the piggyBac superfamily have employed different survival strategies. The transposase-derived gene of the Kobuta subfamily was domesticated before the separation of $X$. tropicalis and $X$. laevis. In contrast, the transposase of the Uribo2 subfamily retained its catalytic activity and can still excise transposons from DNA [9].

Nonautonomous TEs can also be domesticated. Miniature inverted-repeat transposable elements (MITEs) are a subclass of short nonautonomous DNA transposons. These are characterized by a TIR structure, high copy numbers, and highly similar sequences among copies [10]. MITEs generate functional transcriptional regulatory elements [11] and matrix attachment regions [12]. In Xenopus, MITEs also form simple sequence repeats (SSRs). Xmix, a predominant MITE in X. laevis and X. tropicalis, has an amplified internal segment representing a large SSR family, Xstir [13, 14]. Simple sequence repeats are essential for higher-order chromosomal structure [15].

Xmix is a member of the T2 MITE family (T2-MITE), which is characterized by a TTAA target site and a terminal AGGRR (R: A or G) motif in its TIR [16, 17]. These features are common to members of the DNA transposon superfamily Kolobok, which targets the TTAA site and has an RR terminal sequence [18]. Therefore, T2-MITE is presumably a nonautonomous member of the Kolobok DNA transposon superfamily. We classified 16 major T2-MITE subfamilies based on an in silico screening of the $X$. tropicalis genome sequence [19]. Subfamilies A1 (T2-A1, Xmix) and C (T2-C) were the most prevalent and were present in both $X$. tropicalis and X. laevis. They probably originated before these two lineages diverged $\sim 48$ Mya [20]. Despite their age, both subfamilies include "young" (highly homogeneous) members. Therefore, they probably underwent relatively recent amplification. We also found evidence for intraspecific T2-A1 and T2-C insertion polymorphisms [21]. These results suggest that these subfamilies have been actively transposing for more than 48 million years. Their extraordinary longevity suggests that their continued transpositional activity has been conserved by natural selection. The transposition and/or amplification of these subfamilies presumably have been advantageous to the host. We found that the sequences derived from $\mathrm{T} 2-\mathrm{C}$ in $X$. tropicalis were significantly over-represented in the $5^{\prime}$ upstream regions of genes. Therefore, they may regulate the expression of neighboring genes [22].

If the transpositional activities of T2-MITEs are conserved by natural selection, then transposases such as Kolobok, which are presumably responsible for their transposition, should be domesticated by the host. In the present study, we surveyed Kolobok transposase genes in the diploid $X$. tropicalis genome and the recently decoded allotetraploid X. laevis genome [20]. To elucidate their evolution within the hosts, we analyzed their molecular phylogenies, copy numbers, syntenies, and sequence conservation.

\section{Methods}

Search for prospective Kolobok transposase-coding sequences

$X$. tropicnalis (Nigerian 9.1) and X. laevis (J-Strain 9.2) genome assemblies were downloaded from the Xenbase FTP site [23, 24] and used in the analyses described below. An automated pipeline for these analyses was developed using the Ruby language.

The search for prospective Kolobok transposase-coding sequences (CDSs) was carried out as follows. Query Kolobok superfamily transposase protein sequences were collected from vertebrate, zebrafish, and invertebrate 
data sets using Repbase Update [25] v. 22.09 [26] (Additional file 5: Table S1) and applied to a tblastn search (e-value <1e-5) [27]. The hit regions were extracted with flanking sequences (1800 bp each upstream and downstream). The longest open reading frames (ORFs) in each extracted sequence were treated as candidate Kolobok transposase CDSs. ORFs that were too short (< $1800 \mathrm{bp}$ ) were excluded from the following analyses. To confirm the homology of proteins encoded in the candidate CDSs to the Kolobok transposases, the CDSs were translated to amino acid sequences and used as queries in backward homology searches (blastp, e-value $<1 \mathrm{e}-5$ ) to the transposase sequences used as the queries in the forward tblastn search. Candidate CDSs with prospective amino acid sequences that were homologous to at least five Kolobok transposases were selected. The protein sequences predicted from the candidate CDSs were aligned by MEGA7 [28] using MUSCLE [29] as an alignment engine. The positions of their start methionines were verified. Excess $5^{\prime}$ regions were manually trimmed from the candidate CDSs to align the translation initiation site. Trimmed CDSs and protein sequences were used in the analyses described below.

\section{Copy number analysis}

Prospective Kolobok transposase CDSs from $X$. laevis and $X$. tropicalis were used as queries for blastn searches (e-value $<1 \mathrm{e}-100)$ to the corresponding genome. Adjacent high-scoring segment pairs were considered single hit regions if the distance between them was less than the query length. Hit regions in each prospective CDS were counted as closely related truncated copies of the CDS if they did not overlap with any prospective CDSs.

\section{Molecular phylogenetics and synteny analyses}

Prospective Kolobok transposase CDSs from both species were translated to protein sequences and used in molecular phylogenetic analyses. The amino acid sequences were realigned with MEGA7 and the alignment engine MUSCLE and used to construct phylogenetic trees. The neighbor-joining method [30] was used to generate the phylogenetic trees with MEGA7. Positions with less than $60 \%$ site coverage were eliminated. The evolutionary distances were computed using the JTT matrix-based method [31]. Rate variations among sites were modeled with a gamma distribution (shape parameter $=1$ ). Dot plot analyses were performed with the polydot program in EMBOSS [32]. The word sizes for the nucleotide and protein sequences were 12 and 5 , respectively.

Putative orthologous transposase sets were collected with reference to the phylogenetic tree. NCBI gene models (XT9_1_GCA.gff3 and XL9_2_GCA.gff3) were downloaded from the Xenbase FTP site [23, 24]. Gene models around the putative orthologous CDSs were compared. The CDS orthologs were defined as those located on the homologous $X$. tropicalis chromosome and $X$. laevis $\mathrm{L} / \mathrm{S}$ chromosomes and flanked by multiple orthologous neighbor genes.

The ratios of synonymous and nonsynonymous substitutions between two coding DNA sequences $(\mathrm{dN} / \mathrm{dS}$ ratio) were calculated by the method of Yang and Nielsen [33] using the yn00 program of PAML v. 4.8 [34].

\section{Search for full-length Kolobok transposons}

The upstream and downstream sequences flanking the CDSs of each repetitive $X K o l$ subfamily were compared by dot plot analysis and multiple alignment to find the left and right TIRs, respectively. The left and right terminal sequences were compared to confirm their similarity. The sequences between similar left and right TIRs were considered as full-length TEs. Four-base pair sequences flanking the full-length TEs were examined to check the conservation of their duplicated target sites (TTAA).

\section{Cloning of $X K o l$-Tpases from two $X$. tropicalis lineages}

$X$. tropicalis was provided by the National Bioresource Project, Japan [35]. Genomic DNAs were extracted from the Nigerian and Asashima lines using a previously described method [13]. Polymerase chain reaction (PCR) was performed using KOD-Plus DNA polymerase (Toyobo Co. Ltd., Osaka, Japan) and 100 ng genomic DNA. The default PCR conditions were as follows: initial denaturation $\left(94{ }^{\circ} \mathrm{C}, 120 \mathrm{~s}\right)$, followed by 35 cycles of denaturation $\left(98^{\circ} \mathrm{C}, 10 \mathrm{~s}\right)$, annealing $\left(60^{\circ} \mathrm{C}, 30 \mathrm{~s}\right)$, and extension $\left(68{ }^{\circ} \mathrm{C}, 90 \mathrm{~s}\right)$. The annealing temperature, extension time, and/or PCR cycles were changed as needed to optimize amplification. The primers used in the PCR are shown in (Additional file 6: Table S2). PCR products were inserted into the pCRBluntII-TOPO or pCR4Blunt-TOPO vector (Invitrogen, Carlsbad, CA, USA) and cloned into OneShotTOP-10 competent cells (Invitrogen). The cloned sequences were analyzed using a BigDye3.1 Terminator Cycle Sequencing Kit (Applied Biosystems, Foster City, CA, USA) and an ABI PRISM 310 or 3100 Genetic Analyzer (Applied Biosystems).

\section{Results}

Prospective Kolobok transposase CDSs and proteins

To identify candidates of active (able to catalyze TE transposition) or domesticated Kolobok transposase genes, we performed tblastn (protein query versus nucleotide database) homology searches of $X$. tropicalis and $X$. laevis genomes. We searched the hit loci and their surrounding regions for the longest ORFs that encoded proteins homologous to Kolobok transposases. Multiple alignments of putative proteins encoded in the longest ORFs revealed that the $N$-terminal sequences 
encoded by certain ORFs were longer than those of other ORFs. We trimmed the excess 5 ' regions from these ORFs to align their start codons with those of the majority genes (Additional file 1: Figure S1). Figure 1 shows the $N$-terminal region and some conserved regions of the aligned proteins. It is unclear whether these trimmed ORFs were the actual CDSs of Kolobok transposase genes. Nevertheless, we expected that most of these ORFs coincided or overlapped with the CDSs. Therefore, we ascribed them to be CDSs of prospective Kolobok transposase genes. In X. tropicalis and X. laevis, 38 and 24 prospective CDSs were found, respectively. We referred to the prospective Xenopus Kolobok transposons as the XKol family and its transposase as XKol-Tpase. We designated the XKol-Tpase CDSs and proteins serially as $\operatorname{Tr} 1-\operatorname{Tr} 38$ for $X$. tropicalis and Lv1-Lv24 for $X$. laevis (Table 1). The five shortest XKol-Tpase proteins (Tr34-Tr38) lacked a certain number of conserved amino acids (Fig. 1 and Table 2). Therefore, they may not be functional. The transposases encoded in the Kolobok-1_XT and Kolobok-2_XT transposons previously reported in the RepBase [18] most resembled, but were not identical to, $\operatorname{Tr} 8$ (723/783 identical) and $\operatorname{Tr} 7$ (782/783 identical), respectively.

Kolobok superfamily transposases usually possess three motifs: a catalytic "DDE" domain, a THAP DNA-binding domain, and an $\mathrm{H} 2 \mathrm{CH}$ putative zinc-finger domain [18, 36]. The THAP DNA-binding domain includes a $\mathrm{C} 2 \mathrm{CH}$ consensus, three key residues (P, W, and F), and a C-terminal AVPTIF box [37]. These features were identified in the prospective $\mathrm{XKol}$-Tpases (Fig. 1). The $\mathrm{H} 2 \mathrm{CH}$ domain is highly conserved in the prospective XKol-Tpases. Thirty-five of the $38 X K o l$-Tpases in $X$. tropicalis and 22 of the 24 XKol-Tpases in X. laevis retained this motif (Table 2). Three THAP domain motifs were also conserved. The $\mathrm{C} 2 \mathrm{CH}$ motif was conserved in 31 of 38 and 24 of 24 XKol-Tpases in $X$. tropicalis and $X$. laevis, respectively. The PWF motif was conserved in 33 of 38 and 24 of 24 XKol-Tpases in $X$. tropicalis and $X$. laevis, respectively. The AVPTIF motif was conserved only in 17 of 38 and 12 of 24 XKol-Tpases in X. tropicalis and X. laevis, respectively. However, most of the remaining $X K o l$-Tpases had chemically similar residues at this position. When considering such residues as conserved, this motif was conserved in 27 of 38 and 23 of 24 XKol-Tpases in X. tropicalis and X. laevis, respectively. In contrast, the catalytic DDE motif was conserved in only 24 of $38 \mathrm{XKol}$-Tpases in X. tropicalis and seven of $24 \mathrm{XKol}$-Tpases in X. laevis. Some XKol-Tpases lost one or two of these motifs by deletion ( $\operatorname{Tr} 3$, $\operatorname{Tr} 25, \operatorname{Tr} 34, \operatorname{Tr} 35, \operatorname{Tr} 36, \operatorname{Tr} 37$, and $\operatorname{Tr} 38)$. These proteins may lack DNA-binding and/or catalytic activity. In

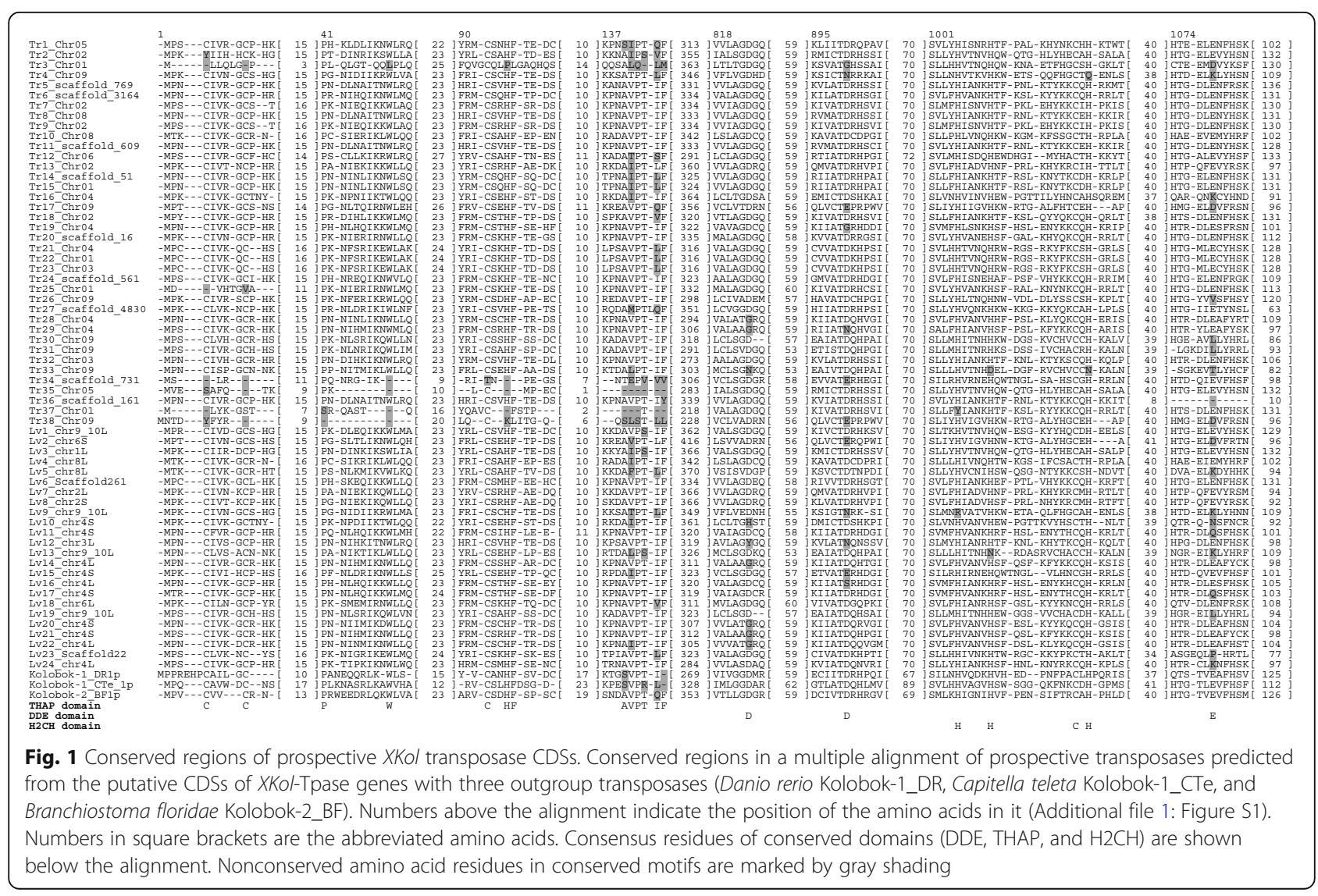


Table 1 Prospective Xenopus XKol transposase CDSs

\begin{tabular}{|c|c|c|c|c|}
\hline Name & Locus & Longest ORF & Trimmed CDS & No. of truncated copies \\
\hline \multicolumn{5}{|c|}{ A. Prospective $X$. tropicalis $X K o l$ transposase CDSs } \\
\hline $\operatorname{Tr} 1$ & Chr05:4592573..4595167(-) & $2595 \mathrm{bp}$ & 2202 bp & 1 \\
\hline $\operatorname{Tr} 2$ & Chr02:100830975..100833458(+) & $2484 \mathrm{bp}$ & $2421 \mathrm{bp}$ & 3 \\
\hline $\operatorname{Tr} 3$ & Chr01:90056839..90059247(+) & $2409 \mathrm{bp}$ & $2409 \mathrm{bp}$ & 0 \\
\hline $\operatorname{Tr} 4$ & Chr09:49289527..49291908(+) & $2382 \mathrm{bp}$ & $2319 \mathrm{bp}$ & 0 \\
\hline $\operatorname{Tr} 5$ & scaffold_769:44126..46486(-) & $2361 \mathrm{bp}$ & $2361 \mathrm{bp}$ & 10 \\
\hline Tr6 & scaffold_3164:2182.4536(-) & $2355 \mathrm{bp}$ & $2355 \mathrm{bp}$ & 5 \\
\hline $\operatorname{Tr} 7$ & Chr02:77614330..77616681(-) & $2352 \mathrm{bp}$ & $2352 \mathrm{bp}$ & 2 \\
\hline $\operatorname{Tr} 8$ & Chr08:54548260..54550611(+) & 2352 bp & 2352 bp & 9 \\
\hline $\operatorname{Tr} 9$ & Chr02:55486066..55488417(+) & $2352 \mathrm{bp}$ & 2352 bp & 2 \\
\hline $\operatorname{Tr} 10$ & Chr08:50277255..50279597(+) & $2343 \mathrm{bp}$ & 2292 bp & 0 \\
\hline $\operatorname{Tr} 11$ & scaffold_609:18293..20635(-) & $2343 \mathrm{bp}$ & $2343 \mathrm{bp}$ & 9 \\
\hline $\operatorname{Tr} 12$ & Chr06:112308032..112310368(-) & $2337 \mathrm{bp}$ & $2250 \mathrm{bp}$ & 9 \\
\hline $\operatorname{Tr} 13$ & Chr02:162067553..162069883(-) & $2331 \mathrm{bp}$ & $2331 \mathrm{bp}$ & 0 \\
\hline $\operatorname{Tr} 14$ & scaffold_51:231432..233759(-) & $2328 \mathrm{bp}$ & $2328 \mathrm{bp}$ & 5 \\
\hline $\operatorname{Tr} 15$ & Chr01:83498100..83500424(+) & $2325 \mathrm{bp}$ & $2325 \mathrm{bp}$ & 5 \\
\hline $\operatorname{Tr} 16$ & Chr04:108160710..108163031(-) & $2322 \mathrm{bp}$ & $2322 \mathrm{bp}$ & 0 \\
\hline $\operatorname{Tr} 17$ & Chr09:29639969..29642278(-) & $2310 \mathrm{bp}$ & $2310 \mathrm{bp}$ & 0 \\
\hline $\operatorname{Tr} 18$ & Chr02:66952233..66954539(-) & $2307 \mathrm{bp}$ & $2307 \mathrm{bp}$ & 0 \\
\hline $\operatorname{Tr} 19$ & Chr04:41679747..41682050(+) & 2304 bp & $2229 \mathrm{bp}$ & 0 \\
\hline $\operatorname{Tr} 20$ & scaffold_16:1256554.1258851(+) & $2298 \mathrm{bp}$ & 2298 bp & 4 \\
\hline $\operatorname{Tr} 21$ & Chr04:107657217..107659511(+) & $2295 \mathrm{bp}$ & $2295 \mathrm{bp}$ & 9 \\
\hline $\operatorname{Tr} 22$ & Chr01:168455606..168457900(-) & $2295 \mathrm{bp}$ & $2295 \mathrm{bp}$ & 9 \\
\hline $\operatorname{Tr} 23$ & Chr03:106168762..106171056(-) & $2295 \mathrm{bp}$ & $2295 \mathrm{bp}$ & 9 \\
\hline $\operatorname{Tr} 24$ & scaffold_561:30795..33086(-) & 2292 bp & $2256 \mathrm{bp}$ & 0 \\
\hline $\operatorname{Tr} 25$ & Chr01:107512548..107514821(+) & 2274 bp & 2274 bp & 4 \\
\hline $\operatorname{Tr} 26$ & Chr09:13318232..13320439(-) & 2208 bp & 2208 bp & 0 \\
\hline $\operatorname{Tr} 27$ & scaffold_4830:88..2292(-) & $2205 \mathrm{bp}$ & $2205 \mathrm{bp}$ & 5 \\
\hline $\operatorname{Tr} 28$ & Chr04:128881775..128883943(-) & $2169 \mathrm{bp}$ & $2169 \mathrm{bp}$ & 0 \\
\hline $\operatorname{Tr} 29$ & Chr04:128872195..128874363(-) & $2169 \mathrm{bp}$ & $2169 \mathrm{bp}$ & 0 \\
\hline $\operatorname{Tr} 30$ & Chr09:10540280..10542436(+) & $2157 \mathrm{bp}$ & $2157 \mathrm{bp}$ & 0 \\
\hline $\operatorname{Tr} 31$ & Chr09:10547870..10549993(+) & $2124 \mathrm{bp}$ & 2091 bp & 0 \\
\hline $\operatorname{Tr} 32$ & Chr03:80573408..80575504(+) & 2097 bp & 2097 bp & 0 \\
\hline $\operatorname{Tr} 33$ & Chr09:10557320..10559413(+) & 2094 bp & 2094 bp & $1^{*}$ \\
\hline $\operatorname{Tr} 34$ & scaffold_731:1892..3937(-) & 2046 bp & 2046 bp & 0 \\
\hline $\operatorname{Tr} 35$ & Chr05:37113502..37115535(-) & 2034 bp & 2034 bp & 3 \\
\hline $\operatorname{Tr} 36$ & scaffold_161:120530..122407(+) & 1878 bp & 1878 bp & 8 \\
\hline $\operatorname{Tr} 37$ & Chr01:130854739..130856610(-) & 1872 bp & 1872 bp & 0 \\
\hline $\operatorname{Tr} 38$ & Chr09:29654976..29656787(-) & 1812 bp & 1812 bp & 0 \\
\hline \multicolumn{5}{|c|}{ B. Prospective $X$. laevis $X K o /$ transposase CDSs } \\
\hline LV1 & chr9_10L:117582522..117585011(-) & $2490 \mathrm{bp}$ & $2433 \mathrm{bp}$ & 4 \\
\hline LV2 & chr6S:1512718..1515198(-) & $2481 \mathrm{bp}$ & $2481 \mathrm{bp}$ & $1^{*}$ \\
\hline LV3 & chr1L:4995471..4997924(-) & $2454 \mathrm{bp}$ & $2454 \mathrm{bp}$ & 4 \\
\hline LV4 & chr8L:50116481..50118844(+) & $2364 \mathrm{bp}$ & 2292 bp & 0 \\
\hline
\end{tabular}


Table 1 Prospective Xenopus XKol transposase CDSs (Continued)

\begin{tabular}{|c|c|c|c|c|}
\hline Name & Locus & Longest ORF & Trimmed CDS & No. of truncated copies \\
\hline LV5 & Scaffold261:26626.28977(+) & $2352 \mathrm{bp}$ & $2352 \mathrm{bp}$ & 0 \\
\hline Lv6 & chr8L:99349197..99351548(-) & $2352 \mathrm{bp}$ & $2352 \mathrm{bp}$ & 2 \\
\hline LV7 & chr2L:167641114..167643453(-) & $2340 \mathrm{bp}$ & $2340 \mathrm{bp}$ & 0 \\
\hline LV8 & chr2S:147754724..147757057(-) & $2334 \mathrm{bp}$ & $2334 \mathrm{bp}$ & 0 \\
\hline Lv9 & chr9_10L:77656066..77658378(+) & $2313 \mathrm{bp}$ & $2313 \mathrm{bp}$ & 0 \\
\hline LV10 & chr4S:91247377..91249686(-) & $2310 \mathrm{bp}$ & $2310 \mathrm{bp}$ & $1^{*}$ \\
\hline LV11 & chr4S:1463674..1465929(-) & $2256 \mathrm{bp}$ & $2217 \mathrm{bp}$ & $1^{*}$ \\
\hline LV12 & chr3L:58143401..58145647(+) & $2247 \mathrm{bp}$ & $2211 \mathrm{bp}$ & 0 \\
\hline LV13 & chr9_10L:110876140..110878383(-) & $2244 \mathrm{bp}$ & $2244 \mathrm{bp}$ & 0 \\
\hline LV14 & chr4L:133759875..133762109(+) & $2235 \mathrm{bp}$ & $2187 \mathrm{bp}$ & 0 \\
\hline LV15 & chr4S:20852192..20854426(-) & $2235 \mathrm{bp}$ & $2235 \mathrm{bp}$ & 0 \\
\hline LV16 & chr4L:26594086..26596320(+) & $2235 \mathrm{bp}$ & $2235 \mathrm{bp}$ & $1^{*}$ \\
\hline LV17 & chr4S:1470489..1472717(-) & $2229 \mathrm{bp}$ & $2229 \mathrm{bp}$ & $2^{*}$ \\
\hline LV18 & chr6L:145404209..145406428(-) & $2220 \mathrm{bp}$ & $2220 \mathrm{bp}$ & 0 \\
\hline LV19 & chr9_10L:110897783..110899978(-) & $2196 \mathrm{bp}$ & $2196 \mathrm{bp}$ & 0 \\
\hline LV20 & chr4S:1 12529046..112531238(-) & $2193 \mathrm{bp}$ & $2193 \mathrm{bp}$ & 0 \\
\hline LV21 & chr4S:112539344..112541533(-) & $2190 \mathrm{bp}$ & $2190 \mathrm{bp}$ & 0 \\
\hline LV22 & chr4L:133769461..133771647(+) & $2187 \mathrm{bp}$ & $2187 \mathrm{bp}$ & 0 \\
\hline LV23 & Scaffold22:1796503..1798647(-) & $2145 \mathrm{bp}$ & $2145 \mathrm{bp}$ & 3 \\
\hline LV24 & chr4L:26633802..26635940(+) & $2139 \mathrm{bp}$ & $2103 \mathrm{bp}$ & 0 \\
\hline
\end{tabular}

Prospective XKol-Tpases found in (A) X. tropicalis and (B) X. laevis. Asterisks in the "No. of truncated copies" column indicate semi-nonrepetitive CDSs

summary, 17 X. tropicalis and seven X. laevis XKol-Tpases retained all the motifs characterizing Kolobok transposase.

\section{Truncated copies of the XKol-Tpase genes}

Transposase genes may be retained in a host either by constant amplification of autonomous elements or by domestication in the host. Amplification of autonomous elements results in the accumulation of multiple intact and/or truncated copies in the genome. Therefore, they would have repetitive (multicopy) transposase genes. In contrast, if a copy of a transposase gene was domesticated by its host, it would be conserved by purifying selection. Its undomesticated relatives would not have been conserved by purifying selection and would have accumulated mutations and diverged from the domesticated copy. Over time, then, there would be no apparent homology between a domesticated transposase gene and its relatives, and the domesticated transposase genes would become nonrepetitive (single copies). Conversely, the nonrepetitiveness of a transposase-related gene may indicate that its host domesticated it long ago. X. laevis is an allotetraploid species retaining two of its progenitor's subgenomes as $\mathrm{L}$ and $\mathrm{S}$ chromosomes, respectively [20]. Therefore, this species could have conserved both homeologous transposases if the genes had been domesticated before the segregation of the two progenitor species. In contrast, if either of the domesticated L or S genes had degraded because of functional redundancy, the remaining prospective gene would be accompanied by a closely related truncated sequence on its homeologous chromosome. If one of the tandemly duplicated paralogous transposase genes was degraded, the remaining gene would be accompanied by a closely related truncated sequence on its neighboring locus. In the analyses discussed below, we describe such genes as "semi-nonrepetitive."

To find nonrepetitive or semi-nonrepetitive genes derived from transposases, we surveyed truncated (nonprospective) copies closely related to each XKol-Tpase CDS using a blastn (nucleotide query versus nucleotide database) search. If a query did not hit genomic sequences other than itself or other XKol-Tpase CDSs, it was considered nonrepetitive. The number of truncated copies and their loci are shown in Table 1 and (Additional file 7: Table S3), respectively. Eighteen of $38 X$. tropicalis XKol-Tpase genes (47\%) and 15 of 24 X. laevis $X K o l$-Tpase genes $(63 \%)$ were found to be nonrepetitive. Two X. laevis XKol-Tpase genes (Lv2 and Lv10) could be considered semi-nonrepetitive because their single truncated copies were located on the homeologous chromosome (Additional file 7: Table S3). One X. laevis XKol-Tpase gene (Lv16) and one X. tropicalis XKol-Tpase gene (Tr33) may also be semi-nonrepetitive, as 
Table 2 Amino acid residues in conserved motifs of Kolobok transposase

\begin{tabular}{|c|c|c|c|c|c|c|}
\hline Name & DDE & $\mathrm{H} 2 \mathrm{CH}$ & $\mathrm{C} 2 \mathrm{CH}$ & PWF & AVPTIF & Conservation \\
\hline $\operatorname{Tr} 1$ & $\mathrm{DDE}$ & $\mathrm{HHCH}$ & $\mathrm{CCCH}$ & PWF & SIPTQF & \\
\hline $\operatorname{Tr} 2$ & DDE & $\mathrm{HHCH}$ & $\mathrm{YCCH}$ & PWF & AIPSVF & \\
\hline $\operatorname{Tr} 3$ & DGD & $\mathrm{HHCH}$ & $-C P$ & PLL & ALQ-LM & \\
\hline $\operatorname{Tr} 4$ & DNK & HHCQ & $\mathrm{CCCH}$ & PWF & ATPTLF & \\
\hline $\operatorname{Tr} 5$ & DDE & $\mathrm{HHCH}$ & $\mathrm{CCCH}$ & PWF & AVPTIF & ++ \\
\hline Tr6 & $\mathrm{DDE}$ & $\mathrm{HHCH}$ & $\mathrm{CCCH}$ & PWF & AVPTIF & ++ \\
\hline $\operatorname{Tr} 7$ & DDE & $\mathrm{HHCH}$ & $\mathrm{CCCH}$ & PWF & AVPTIF & ++ \\
\hline $\operatorname{Tr} 8$ & $\mathrm{DDE}$ & $\mathrm{HHCH}$ & $\mathrm{CCCH}$ & PWF & AVPTIF & ++ \\
\hline $\operatorname{Tr} 9$ & DDE & $\mathrm{HHCH}$ & $\mathrm{CCCH}$ & PWF & AVPTIF & ++ \\
\hline $\operatorname{Tr} 10$ & DDE & $\mathrm{HHCH}$ & $\mathrm{CCCH}$ & PWF & AVPTIF & ++ \\
\hline $\operatorname{Tr} 11$ & DDE & $\mathrm{HHCH}$ & $\mathrm{CCCH}$ & PWF & AVPTIF & ++ \\
\hline $\operatorname{Tr} 12$ & $\mathrm{DDE}$ & $\mathrm{HHCH}$ & $\mathrm{CCCH}$ & PWF & ATPTSF & \\
\hline $\operatorname{Tr} 13$ & DDE & $\mathrm{HHCH}$ & $\mathrm{CCCH}$ & PWF & AIPTLF & + \\
\hline $\operatorname{Tr} 14$ & DDE & $\mathrm{HHCH}$ & $\mathrm{CCCH}$ & PWF & AIPTLF & + \\
\hline $\operatorname{Tr} 15$ & DDE & $\mathrm{HHCH}$ & $\mathrm{CCCH}$ & PWF & AIPTLF & + \\
\hline $\operatorname{Tr} 16$ & DDK & $\mathrm{HHCH}$ & $\mathrm{CCCH}$ & PWF & AIPTIF & \\
\hline $\operatorname{Tr} 17$ & DED & $\mathrm{HHCH}$ & $\mathrm{CCCH}$ & PWF & AVPTQF & \\
\hline $\operatorname{Tr} 18$ & DDE & $\mathrm{HHCH}$ & $\mathrm{CCCH}$ & PWF & AVPTVF & + \\
\hline $\operatorname{Tr} 19$ & DGE & $\mathrm{HHCH}$ & $\mathrm{CCCH}$ & PWF & AVPTIF & \\
\hline $\operatorname{Tr} 20$ & DDE & $\mathrm{HHCH}$ & $\mathrm{CCCH}$ & PWF & AVPTIF & ++ \\
\hline $\operatorname{Tr} 21$ & $\mathrm{DDE}$ & $\mathrm{HHCH}$ & $\mathrm{CCCH}$ & PWF & AVPTLF & + \\
\hline $\operatorname{Tr} 22$ & DDE & $\mathrm{HHCH}$ & $\mathrm{CCCH}$ & PWF & AVPTLF & + \\
\hline $\operatorname{Tr} 23$ & DDE & $\mathrm{HHCH}$ & $\mathrm{CCCH}$ & PWF & AVPTLF & + \\
\hline $\operatorname{Tr} 24$ & DDE & $\mathrm{HHCH}$ & $\mathrm{CCCH}$ & PWF & AVPTIF & ++ \\
\hline $\operatorname{Tr} 25$ & DDE & $\mathrm{HHCH}$ & $-\mathrm{VCH}$ & PWF & AVPTIF & \\
\hline $\operatorname{Tr} 26$ & DDV & $\mathrm{HHCH}$ & $\mathrm{CCCH}$ & PWF & AVPTIF & \\
\hline $\operatorname{Tr} 27$ & DDE & $\mathrm{HHCH}$ & $\mathrm{CCCH}$ & PWF & AMPTQF & \\
\hline Tr28 & GDE & $\mathrm{HHCH}$ & $\mathrm{CCCH}$ & PWF & AVPTIF & \\
\hline $\operatorname{Tr} 29$ & GNE & $\mathrm{HHCH}$ & $\mathrm{CCCH}$ & PWF & AVPTIF & \\
\hline $\operatorname{Tr} 30$ & $\mathrm{DDL}$ & $\mathrm{HHCH}$ & $\mathrm{CCCH}$ & PWF & AVPTIF & \\
\hline $\operatorname{Tr} 31$ & $\mathrm{DDL}$ & $\mathrm{HHCH}$ & $\mathrm{CCCH}$ & PWF & AVPTIF & \\
\hline $\operatorname{Tr} 32$ & DDE & $\mathrm{HHCH}$ & $\mathrm{CCCH}$ & PWF & AVPTIF & ++ \\
\hline $\operatorname{Tr} 33$ & NDT & $\mathrm{HDCN}$ & $\mathrm{CCCH}$ & PWF & ALPTIF & \\
\hline $\operatorname{Tr} 34$ & DEE & $\mathrm{HHCH}$ & $-\mathrm{T}-$ & $P_{-}$ & TEPWW & \\
\hline Tr35 & DDE & $\mathrm{HHCH}$ & S-C- & $\mathrm{P}_{-}$ & - & \\
\hline Tr36 & DD- & $\mathrm{HHCH}$ & $\mathrm{CCCH}$ & PWF & AVPTIY & \\
\hline $\operatorname{Tr} 37$ & DDE & $\mathrm{YHCH}$ & -SC- & S-F & $-\mathrm{T}-$ & \\
\hline $\operatorname{Tr} 38$ & DED & $\mathrm{HHCH}$ & Y-CK & $-\mathrm{L}$ & SLSTLL & \\
\hline LV1 & DDE & $\mathrm{HHCH}$ & $\mathrm{CCCH}$ & PWF & AVPSIF & + \\
\hline LV2 & DED & $\mathrm{HHCH}$ & $\mathrm{CCCH}$ & PWF & AVPTLF & \\
\hline LV3 & DDE & $\mathrm{HHCH}$ & $\mathrm{CCCH}$ & PWF & AIPSIF & + \\
\hline LV4 & DDE & $\mathrm{HHCH}$ & $\mathrm{CCCH}$ & PWF & AIPTIF & + \\
\hline
\end{tabular}

Table 2 Amino acid residues in conserved motifs of Kolobok transposase (Continued)

\begin{tabular}{|c|c|c|c|c|c|c|}
\hline Name & DDE & $\mathrm{H} 2 \mathrm{CH}$ & $\mathrm{C} 2 \mathrm{CH}$ & PWF & AVPTIF & Conservation \\
\hline Lv5 & DDK & $\mathrm{HHCH}$ & $\mathrm{CCCH}$ & PWF & AFPTLF & \\
\hline Lv6 & DDE & $\mathrm{HHCH}$ & $\mathrm{CCCH}$ & PWF & AVPTIF & ++ \\
\hline LV7 & DDE & $\mathrm{HHCH}$ & $\mathrm{CCCH}$ & PWF & AVPTIF & ++ \\
\hline LV8 & DDE & $\mathrm{HHCH}$ & $\mathrm{CCCH}$ & PWF & AVPTIF & ++ \\
\hline LV9 & DNK & $\mathrm{RHCH}$ & $\mathrm{CCCH}$ & PWF & ATPTLF & \\
\hline LV10 & $\mathrm{HDN}$ & $\mathrm{HHCH}$ & $\mathrm{CCCH}$ & PWF & AIPTIF & \\
\hline Lv11 & DDQ & $\mathrm{HHCH}$ & $\mathrm{CCCH}$ & PWF & AVPTIF & \\
\hline LV12 & YNE & $\mathrm{HHCH}$ & $\mathrm{CCCH}$ & PWF & AVPTIF & \\
\hline LV13 & DDK & $\mathrm{HNCH}$ & $\mathrm{CCCH}$ & PWF & ALPSIF & \\
\hline Lv14 & GDE & $\mathrm{HHCH}$ & $\mathrm{CCCH}$ & PWF & AVPTIF & \\
\hline Lv15 & DEE & $\mathrm{HHCH}$ & $\mathrm{CCCH}$ & PWF & AIPTIF & \\
\hline Lv16 & DSE & $\mathrm{HHCH}$ & $\mathrm{CCCH}$ & PWF & AVPTIF & \\
\hline Lv17 & DDQ & $\mathrm{HHCH}$ & $\mathrm{CCCH}$ & PWF & AVPTIF & \\
\hline LV18 & DDE & $\mathrm{HHCH}$ & $\mathrm{CCCH}$ & PWF & AVPTVF & + \\
\hline Lv19 & $\mathrm{DDL}$ & $\mathrm{HHCH}$ & $\mathrm{CCCH}$ & PWF & AVPTIF & \\
\hline LV20 & GDE & $\mathrm{HHCH}$ & $\mathrm{CCCH}$ & PWF & AVPTIF & \\
\hline LV21 & GDE & $\mathrm{HHCH}$ & $\mathrm{CCCH}$ & PWF & AVPTIF & \\
\hline LV22 & GDE & $\mathrm{HHCH}$ & $\mathrm{CCCH}$ & PWF & AIPTIF & \\
\hline LV23 & DDP & $\mathrm{HHCH}$ & $\mathrm{CCCH}$ & PWF & AVPTLF & \\
\hline LV24 & DDK & $\mathrm{HHCH}$ & $\mathrm{CCCH}$ & PWF & AVPTIF & \\
\hline
\end{tabular}

The " ++ " in the "Conservation" column indicates that all motifs are conserved. The " + " in the same column indicates that all motifs are conserved except for substitutions of similar amino acids in the AVPTIF motif

their hit sequences were located in the neighborhood of the XKol-Tpase CDSs and may therefore have been amplified by tandem duplication rather than transposition. Lv11 and Lv17 had one and two truncated hits, respectively. However, one of their hit regions covered the entire length of a very short Scaffold105792 (216 bp). Therefore, this hit could be an artifact caused by an imperfect genome sequence assembly. Another hit sequence to Lv17 was located on the neighbor of its homeolog Lv16 and might be a truncated paralog of Lv16 (Additional file 7: Table S3 and Fig. 4). Lv11 and Lv17, then, may also be semi-nonrepetitive genes. It is possible that the host domesticated these nonrepetitive or semi-nonrepetitive genes long ago.

\section{Phylogenetic analyses of XKol-Tpases}

If a transposase gene was domesticated before the divergence of $X$. laevis and $X$. tropicalis and is conserved in both species, then the $X$. laevis and $X$. tropicalis genes are orthologous and the two $X$. laevis genes are homeologous. They should be closely related in the molecular phylogenetic tree and located on homologous chromosome loci. The $\mathrm{L}$ and $\mathrm{S}$ genes derived from ancestral $\mathrm{L}$ and $\mathrm{S}$ species are located on the $\mathrm{L}$ and $\mathrm{S}$ chromosomes, 
respectively. Therefore, if both were conserved in X. laevis, then the branching pattern should be a triplet consisting of the $X$. tropicalis (semi-)nonrepetitive transposase and the two (semi-)nonrepetitive transposases on the $X$. laevis $\mathrm{L}$ and $\mathrm{S}$ chromosomes (triplet-branching). Alternatively, if either the L or S gene was lost or degraded in $X$. laevis, the branching pattern should consist of a pair of (semi-)nonrepetitive transposases from $X$. tropicalis and $X$. laevis (doublet-branching).

Figure 2 shows a molecular phylogenetic tree for the $X K o l$-Tpases. A typical triplet-branching pattern can be seen for nonrepetitive Tr13, Lv7, and Lv8 (grouped as subfamily "D1" in Figs. 2 and 3), whose genes are located on the homologous chromosomes $X$. tropicalis Chr02, $X$. laevis chr2L, and chr2S, respectively. X. tropicalis $\operatorname{Tr} 13$ branched first, followed by the branching of $X$. laevis Lv7 and Lv8. This pattern corresponded to the order of divergence in $X$. tropicalis and two ancestral species of $X$. laevis. A dot plot analysis showed that their genes were significantly similar in amino acid and nucleotide sequences (Fig. 3). This relationship could also be seen for the other three (semi-)nonrepetitive XKol-Tpase triplets: Tr19/Lv16/Lv11-Lv17 (D2), Tr28/Lv22/Lv20 (D3-1), and Tr29/Lv14/Lv21 (D3-2). Lv11 and Lv17 genes were tandemly located within $\sim 4.6 \mathrm{~kb}$ of each other on chr4S. Lv16 was found to be a semi-nonrepetitive gene accompanied by a closely related truncated sequence located in its neighborhood (Fig. 4). Therefore, these genes were thought to be tandemly duplicated before the segregation of the two ancestor species of $X$. laevis. The last two triplets Tr28/Lv22/Lv20 and Tr29/Lv14/Lv21 were closely related (Fig. 2). The tandemly duplicated gene pairs Tr28-Tr29, Lv14-Lv22, and Lv2-Lv20 were located within $\sim 8 \mathrm{~kb}$ of each other on the homologous chromosomes $X$. tropicalis Chr04, X. laevis chr4L, and chr4S, respectively (Fig. 4). These paired genes were thought to be paralogs tandemly duplicated before the divergence of $X$. laevis and $X$. tropicalis. Six doublet pairs of closely related $X$. tropicalis and X. laevis (semi-)nonrepetitive genes were found to be located on the homologous chromosomes $\operatorname{Tr} 16 / \mathrm{Lv} 10$ (chr4, D4), Tr10/Lv4 (chr8, D5), Tr30/Lv19 (chr9, D6-1), Tr33/Lv13 (chr9, D6-2), Tr4/Lv9 (chr9, D7), and Tr32/ Lv12 (chr3, D8). One doublet pair, Tr34/Lv15 (D9), was also closely related; however, the chromosomal location of Tr34 was uncertain. These pairs may also be orthologs domesticated by the common ancestor of $X$. laevis and $X$. tropicalis. One of the $X$. laevis homeologs may have been lost or broken.

Some X. tropicalis XKol-Tpase genes encode highly similar proteins and are located on different chromosomes. These genes were probably recently amplified. For example, Tr21, Tr22, and Tr23 (grouped as subfamily "A1" in Figs. 2 and 3) were found to be located on Chr04, Chr01, and Chr03, respectively (Tables 1 and 3,
Figs. 2 and 3). They also contained multiple truncated copies of XKol-Tpase genes on Chr01, Chr02, Chr04, Chr05, Chr06, and Chr09 (Additional file 7: Table S3). Another five $X$. tropicalis XKol-Tpase gene groups, i.e., Tr5-Tr8-Tr11-Tr36 (A2), Tr14-Tr15 (A3), Tr20-Tr25 (A4), Tr2-Tr35 (A5), and Tr7-Tr9 (A6), were also closely related to each other and had multiple truncated copies on different chromosomes (Fig. 2, Additional file 7: Table S3). Although $X$. tropicalis $\operatorname{Tr} 6, \operatorname{Tr} 12$, and $\operatorname{Tr} 27$ and X. laevis Lv1, Lv3, Lv6, and Lv23 did not have similar XKol-Tpase genes, they had truncated copies on various chromosomes (Additional file 7: Table S3), representing traces of their amplification and transposition. Similarities in nucleotide sequences between these $X K o l$-Tpase genes and their truncated hits are shown by dot plots (Additional file 2: Figure S2).

\section{Evidence of $X K \mathrm{Kol}$-Tpase gene domestication}

The triplet or doublet XKol-Tpase genes described above were thought to have been domesticated by the common ancestor of Xenopus. We analyzed the neighbors of these putative domesticated XKol-Tpases to assess whether the genes in each triplet or doublet were located on homologous loci. All four triplets (D1, D2, D3-1, and D3-2) and five doublets (D4, D5, D6-1, D6-2, and D7) in the X. tropicalis and $X$. laevis genes conserved synteny with the neighbors (Fig. 4). In contrast, we did not find synteny for the doublet pairs D8 and D9 (data not shown).

We calculated the $\mathrm{dN} / \mathrm{dS}$ ratio between putative orthologous or similar X. tropicalis and X. laevis XKol-Tpase genes to determine whether they were affected by natural selection (Table 4B). All dN/dS ratios were less than 1.0; therefore, these genes were probably conserved by purifying selection.

We investigated the evolutionary conservation of the CDSs compared to their flanking $2000 \mathrm{bp}$ sequences for subfamilies D1-D9 (Additional file 3: Figure S3). The dot plots showed that the CDS regions were more conserved than their flanking regions. This result also supported the hypothesis that the XKol-Tpase proteins have a function conserved by natural selection.

We amplified and cloned the CDSs of the putative domesticated $\mathrm{XKol}$-Tpase genes from the distinct Nigerian and Asashima lineages of $X$. tropicalis. There were nucleotide substitutions and indels between the clones of the two lines. Nevertheless, the ORFs of the CDSs were not destroyed by nonsense or frameshift mutations (Table 4A and Additional file 4: Figure S4). For the $X$. tropicalis triplet and doublet genes, the $\mathrm{dN} / \mathrm{dS}$ ratios between the Nigerian and Asashima lines were less than 1.0 in all cases except for $\operatorname{Tr} 19$, wherein all four substitutions were nonsynonymous. We also cloned and compared CDSs from nine other nonrepetitive genes ( $\operatorname{Tr} 3$, Tr17, $\operatorname{Tr} 18, \operatorname{Tr} 24, \operatorname{Tr} 26, \operatorname{Tr} 31, \operatorname{Tr} 32, \operatorname{Tr} 34$, and $\operatorname{Tr} 38)$ in 


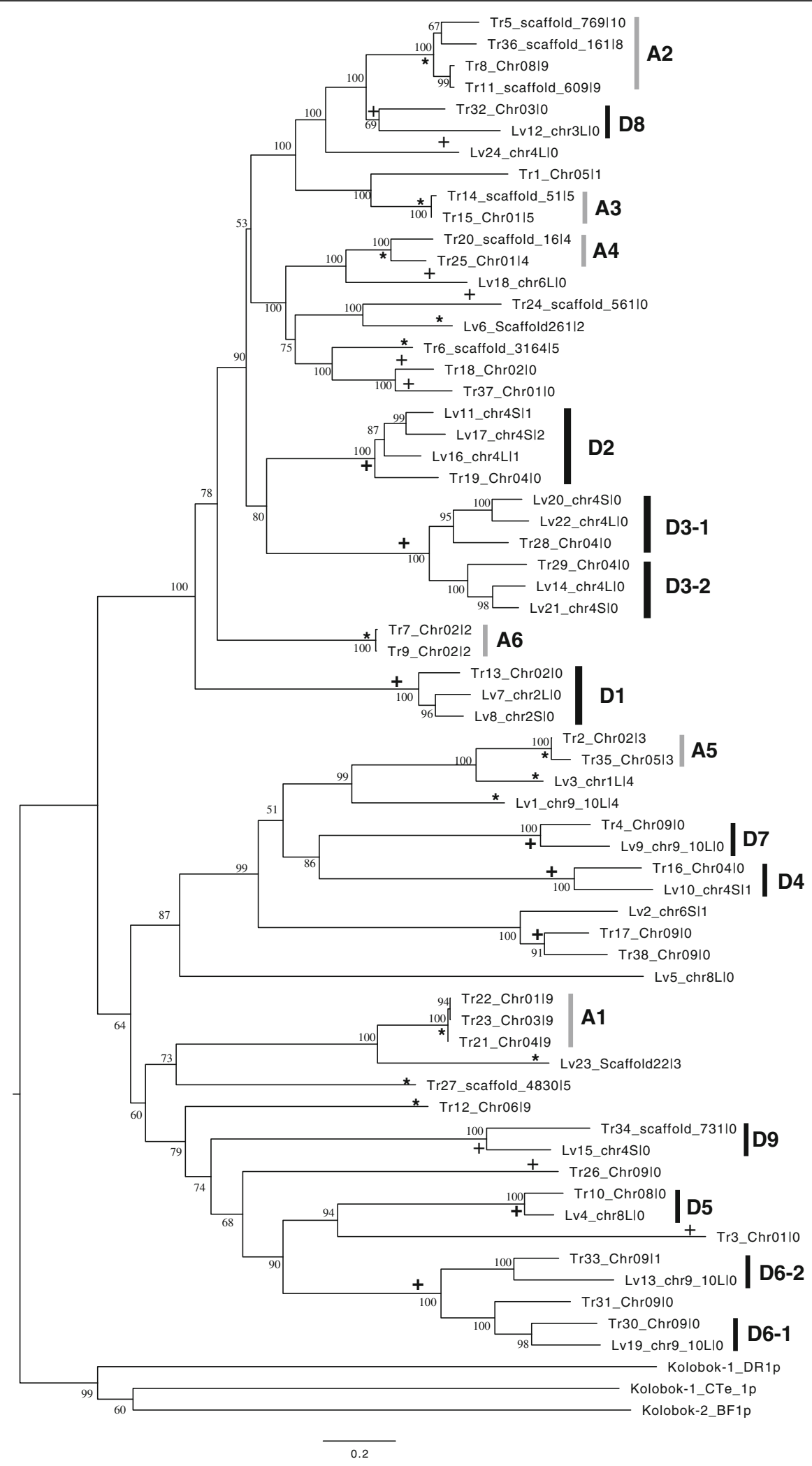

Fig. 2 (See legend on next page.) 
(See figure on previous page.)

Fig. 2 Molecular phylogeny of prospective XKol transposases. Molecular phylogenetic trees of XKol-Tpase amino acid sequences inferred using the neighbor-joining method. Each operational taxonomic unit is represented by the names of the CDS and a chromosome or scaffold on which the CDS is located. The number of blastn hits to truncated transposase sequences are shown as numerals following the vertical bar. The percentage of replicate trees in which the associated taxa clustered together in the bootstrap test (500 replicates) are shown next to the branches. The symbol " + " on the branches indicates domestication events. Asterisks indicate transpositional amplification events. Subfamilies of putative domesticated transposase orthologs are grouped by black bars and named D1-D9. CDS subfamilies that may have been duplicated by recent transposition and amplification are grouped by gray bars and designated A1-A6

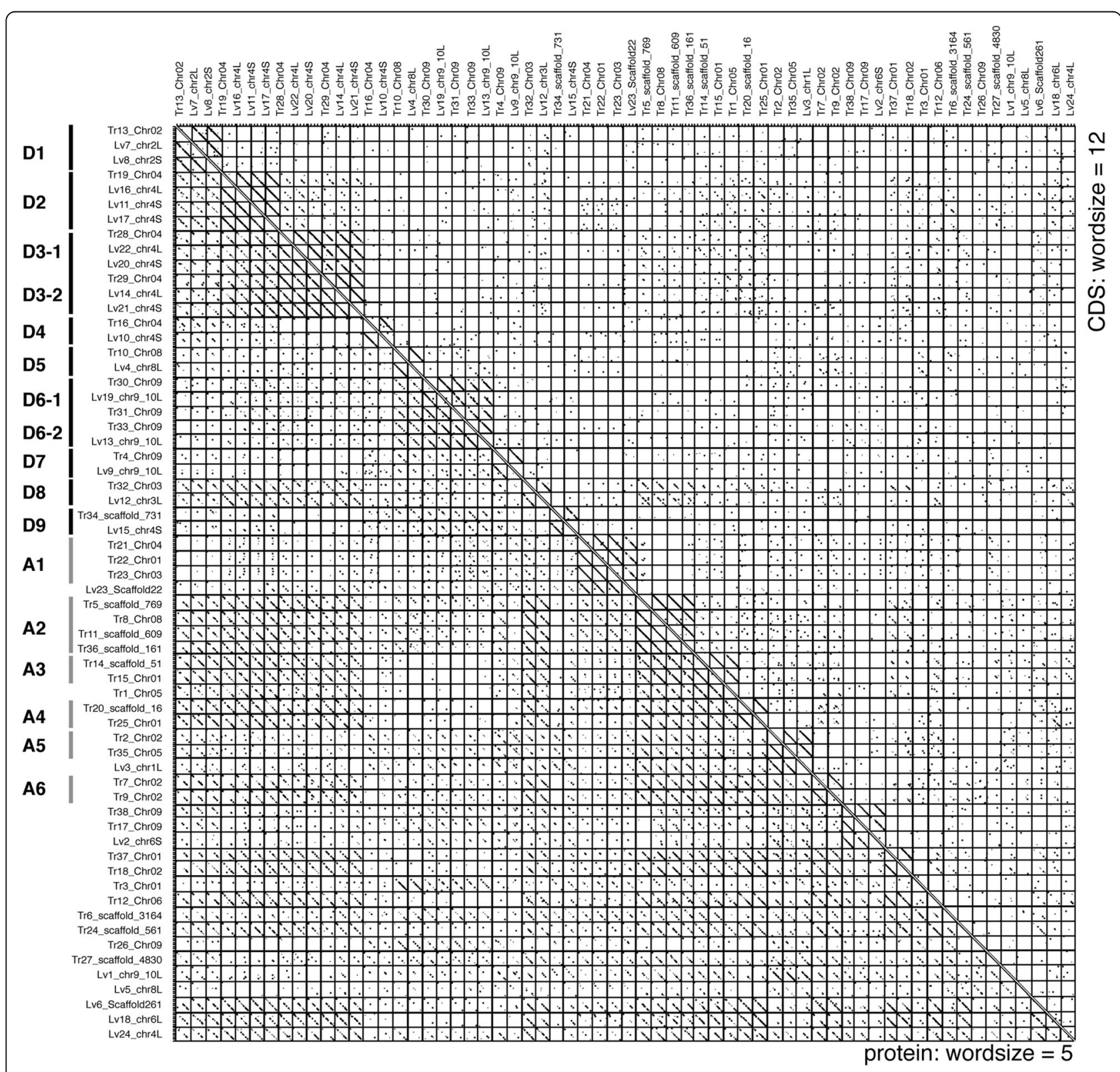

Fig. 3 Dot plot analysis of XKol-Tpase gene CDSs. All-to-all comparisons of XKol-Tpase CDSs (upper right) and proteins (lower left) performed by dot plot analyses. D1-D9 and A1-A6 are grouped in the same way as in Fig. 2 
D1

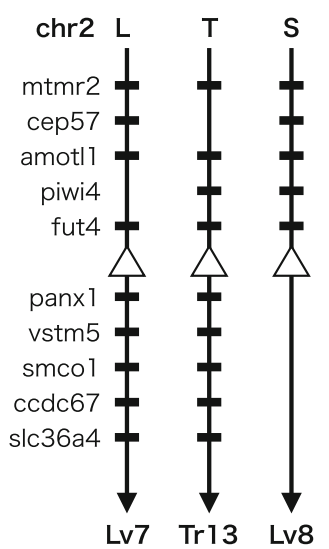

D4

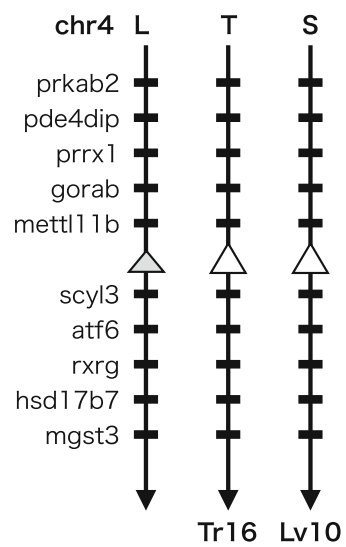

D2

D3

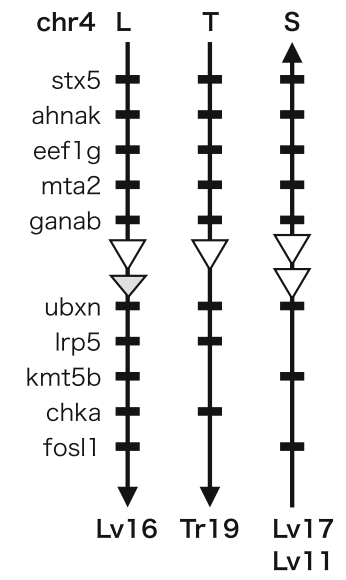

D6



D7
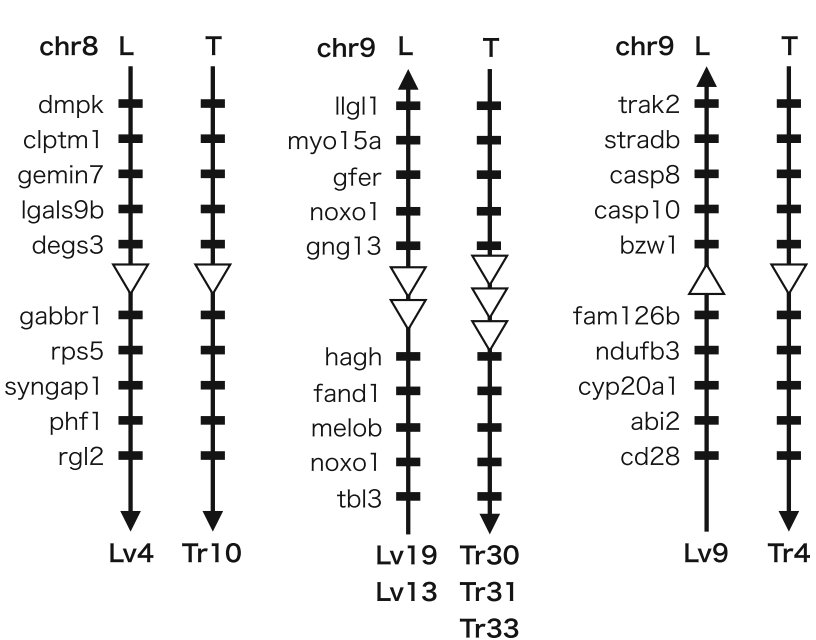

Tr33

Fig. 4 Synteny of XKol-Tpase genes. Conserved synteny around putative domesticated orthologs of subfamily D1-D7. Xenopus laevis homeologous chromosomes $L$ and $S$ and Xenopus tropicalis homologous chromosome $(T$ ) are indicated by arrows. The orientation of each arrow indicates the 5' to 3' direction. XKol-Tpase CDSs are represented by white triangles on the chromosomes, and their names are shown below the chromosomes. Gray triangles indicate truncated XKol-Tpase sequences. Gene models around the CDSs are represented by black bars

the Nigerian and Asashima lines (Table 4A and Additional file 4: Figure S4). Once again, neither substitutions nor indel mutations destroyed the ORFs. The $\mathrm{dN} /$ dS ratios were $<1.0$ for seven genes, approximately 1.0 for $\operatorname{Tr} 3$, and $>1.0$ for $\operatorname{Tr} 32$. These results indicated that most nonrepetitive or semi-nonrepetitive XKol-Tpase genes were domesticated by the host and conserved by purifying selection.

\section{Conservation of repetitive and recently active XKol-Tpase genes}

In general, active transposons were not conserved by purifying selection because they were untamed and potentially harmful to the host. $X$. tropicalis Tr21-Tr22-Tr23 (A1), Tr5-Tr8-Tr11-Tr36 (A2), Tr14-Tr15 (A3), Tr20-Tr25
(A4), $\operatorname{Tr} 2-\operatorname{Tr} 35$ (A5), $\operatorname{Tr} 7-\operatorname{Tr} 9$ (A6), $\operatorname{Tr} 6, \operatorname{Tr} 12$, and $\operatorname{Tr} 27$ had multiple intact and/or truncated copies. Dot plot analyses revealed that all intact genes and some truncated copies were highly similar (Additional file 2: Figure S2), suggesting that they were recently amplified. Therefore, they were either currently active or were active until recently.

We cloned and compared the CDSs of the repetitive XKol-Tpases in the Nigerian and Asashima lines (Table 4A). The ORFs of $\operatorname{Tr} 22, \operatorname{Tr} 8, \operatorname{Tr} 15, \operatorname{Tr} 20, \operatorname{Tr} 6$, and $\operatorname{Tr} 27$ were conserved and not destroyed by nonsense or frameshift mutations. The clones of $\operatorname{Tr} 22$ and $\operatorname{Tr} 15$ from the Asashima line were identical to those from the Nigerian line. For $\operatorname{Tr} 2$ and $\operatorname{Tr} 12$, frameshift mutations destroyed the ORFs of the 
Table 3 Relationship of transposases between $X$. tropicalis and $X$. laevis

\begin{tabular}{|c|c|c|c|}
\hline Name & Closely related CDS & Subfamily & Synteny \\
\hline \multicolumn{4}{|c|}{ A. Relationship between $X$. tropicalis and $X$. laevis transposases } \\
\hline \multicolumn{4}{|l|}{$\operatorname{Tr} 1$} \\
\hline $\operatorname{Tr} 2$ & $\operatorname{Lv} 3(L)$ & A5 & \\
\hline \multicolumn{4}{|l|}{$\operatorname{Tr} 3$} \\
\hline $\operatorname{Tr} 4$ & $\operatorname{Lv} 9(L)$ & D7 & + \\
\hline $\operatorname{Tr} 5$ & & A2 & \\
\hline \multicolumn{4}{|l|}{ Tr6 } \\
\hline $\operatorname{Tr} 7$ & & A6 & \\
\hline $\operatorname{Tr} 8$ & & A2 & \\
\hline $\operatorname{Tr} 9$ & & A6 & \\
\hline $\operatorname{Tr} 10$ & $\operatorname{Lv} 4(\mathrm{~L})$ & D5 & + \\
\hline $\operatorname{Tr} 11$ & & A2 & \\
\hline \multicolumn{4}{|l|}{$\operatorname{Tr} 12$} \\
\hline $\operatorname{Tr} 13$ & $\operatorname{Lv} 7(\mathrm{~L}), \operatorname{Lv} 8(S)$ & D1 & + \\
\hline $\operatorname{Tr} 14$ & & A3 & \\
\hline $\operatorname{Tr} 15$ & & A3 & \\
\hline $\operatorname{Tr} 16$ & Lv10(S) & D4 & + \\
\hline $\operatorname{Tr} 17$ & $\operatorname{Lv} 2(S)$ & & \\
\hline \multicolumn{4}{|l|}{$\operatorname{Tr} 18$} \\
\hline $\operatorname{Tr} 19$ & LV16(L), LV11(S), LV17(S) & D2 & + \\
\hline Tr20 & & A4 & \\
\hline $\operatorname{Tr} 21$ & LV23 & $\mathrm{A} 1$ & \\
\hline $\operatorname{Tr} 22$ & LV23 & A1 & \\
\hline $\operatorname{Tr} 23$ & LV23 & A1 & \\
\hline \multicolumn{4}{|l|}{$\operatorname{Tr} 24$} \\
\hline $\operatorname{Tr} 25$ & & A4 & \\
\hline \multicolumn{4}{|l|}{ Tr26 } \\
\hline \multicolumn{4}{|l|}{ Tr27 } \\
\hline $\operatorname{Tr} 28$ & Lv22(L), Lv20(S) & D3-1 & + \\
\hline $\operatorname{Tr} 29$ & Lv14(L), LV21(S) & D3-2 & + \\
\hline $\operatorname{Tr} 30$ & Lv19(L) & D6-1 & + \\
\hline $\operatorname{Tr} 31$ & & D6-1 & + \\
\hline $\operatorname{Tr} 32$ & Lv12(L) & D8 & \\
\hline $\operatorname{Tr} 33$ & LV13(L) & D6-2 & + \\
\hline $\operatorname{Tr} 34$ & $\operatorname{Lv15(L)}$ & D9 & \\
\hline $\operatorname{Tr} 35$ & $\operatorname{Lv} 3(L)$ & A5 & \\
\hline $\operatorname{Tr} 36$ & & $A 2$ & \\
\hline \multicolumn{4}{|l|}{$\operatorname{Tr} 37$} \\
\hline $\operatorname{Tr} 38$ & $\operatorname{Lv} 2(S)$ & & \\
\hline \multicolumn{4}{|c|}{ B. Relationship between $X$. laevis and X. tropicalis transposases } \\
\hline \multicolumn{4}{|l|}{ LV1 } \\
\hline LV2 & $\operatorname{Tr} 17^{*}, \operatorname{Tr} 38$ & & \\
\hline
\end{tabular}

Table 3 Relationship of transposases between $X$. tropicalis and $X$. laevis (Continued)

\begin{tabular}{|c|c|c|c|}
\hline Name & Closely related CDS & Subfamily & Synteny \\
\hline LV3 & $\operatorname{Tr} 2 *, \operatorname{Tr} 35$ & & \\
\hline LV4 & $\operatorname{Tr} 10$ & D5 & + \\
\hline \multicolumn{4}{|l|}{ Lv5 } \\
\hline \multicolumn{4}{|l|}{ Lv6 } \\
\hline LV7 & $\operatorname{Tr} 13$ & D1 & + \\
\hline Lv8 & $\operatorname{Tr} 13$ & D1 & + \\
\hline LV9 & $\operatorname{Tr} 4$ & D7 & + \\
\hline LV10 & $\operatorname{Tr} 16$ & D4 & + \\
\hline LV11 & $\operatorname{Tr} 19$ & D2 & + \\
\hline LV12 & $\operatorname{Tr} 32$ & D8 & \\
\hline LV13 & $\operatorname{Tr} 33$ & D6-2 & + \\
\hline LV14 & $\operatorname{Tr} 29$ & D3-2 & + \\
\hline LV15 & $\operatorname{Tr} 34$ & D9 & \\
\hline LV16 & $\operatorname{Tr} 19$ & D2 & + \\
\hline LV17 & $\operatorname{Tr} 19$ & D2 & + \\
\hline \multicolumn{4}{|l|}{ Lv18 } \\
\hline LV19 & $\operatorname{Tr} 30$ & D6-1 & + \\
\hline LV20 & $\operatorname{Tr} 28$ & D3-1 & + \\
\hline LV21 & $\operatorname{Tr} 29$ & D3-2 & + \\
\hline LV22 & $\operatorname{Tr} 28$ & D3-1 & + \\
\hline LV23 & $\operatorname{Tr} 22^{*}, \operatorname{Tr} 21, \operatorname{Tr} 23$ & & \\
\hline LV24 & & & \\
\hline
\end{tabular}

(A) Relationship between $X$. tropicalis and $X$. laevis transposases and (B) relationship between $X$. laevis and $X$. tropicalis transposases. Asterisks in the "closely related CDS" column indicate CDSs used in the analysis of differences in nucleotides and amino acids between X. laevis and X. tropicalis (Table 4B)

clones from the Asashima line. Tr12 from the Asashima line was degraded by many mutations.

We searched the upstream and downstream flanking sequences of the CDSs of the repetitive XKol-Tpases for left and right TIRs and found both left and right ones for ten CDSs (Table 5). All of them had AG terminal sequences, and all except for the right TIR of Tr11 were flanked by an intact TTAA target sequence. These are probably full-length autonomous $X K o l$ copies that still have transposition activity.

\section{Discussion}

Multiple massive domestication of Kolobok transposases

In the present study, we surveyed the $X$. tropicalis and $X$. laevis genomes and found 38 and 24 prospective Kolobok transposase genes, respectively, whose CDSs encoded proteins of more than 600 amino acids in length. Subfamily A2 (Tr8/Tr11) and A6 (Tr7/Tr9) resembled previously reported $X$. tropicalis transposases of Kolobok-1_XT and Kolobok-2_XT, respectively [18], but others were novel genes. It is possible that some of these 
Table 4 Differences in transposase CDSs and amino acids, and presumptive status

\begin{tabular}{|c|c|c|c|c|c|}
\hline Name & Nucleotide substitution & Nucleotide gap & Amino acid substitution & $\mathrm{dN} / \mathrm{dS}$ & Presumptive status \\
\hline \multicolumn{6}{|c|}{ A. Differences between Nigerian and Asashima lines, and presumptive status } \\
\hline $\operatorname{Tr} 1$ & 20 & 6 & 10 & 0.396 & active? \\
\hline $\operatorname{Tr} 2$ & 11 & 24 & frame-shift & - & active? \\
\hline $\operatorname{Tr} 3$ & 5 & 0 & 3 & 1.006 & domestication? \\
\hline $\operatorname{Tr} 4$ & 11 & 12 & 7 & 0.501 & ancient domestication \\
\hline $\operatorname{Tr} 5$ & & & & & currently active \\
\hline $\operatorname{Tr} 6$ & 1 & 0 & 1 & $d S=0$ & currently active \\
\hline $\operatorname{Tr} 7$ & 9 & 0 & 7 & 0.696 & currently active \\
\hline $\operatorname{Tr} 8$ & 26 & 0 & 21 & 1.057 & currently active \\
\hline $\operatorname{Tr} 9$ & & & & & currently active \\
\hline $\operatorname{Tr} 10$ & 5 & 0 & 1 & 0.084 & ancient domestication \\
\hline $\operatorname{Tr} 11$ & & & & & currently active \\
\hline $\operatorname{Tr} 12$ & 205 & 991 & frame-shift & - & active? \\
\hline $\operatorname{Tr} 13$ & 26 & 0 & 8 & 0.173 & ancient domestication \\
\hline $\operatorname{Tr} 14$ & & & & & currently active \\
\hline $\operatorname{Tr} 15$ & 0 & 3 & 0 & $d S=0$ & currently active \\
\hline $\operatorname{Tr} 16$ & 3 & 0 & 2 & 0.527 & ancient domestication \\
\hline $\operatorname{Tr} 17$ & 24 & 0 & 13 & 0.434 & domestication? \\
\hline $\operatorname{Tr} 18$ & 15 & 0 & 9 & 0.504 & domestication? \\
\hline $\operatorname{Tr} 19$ & 4 & 30 & 4 & $d S=0$ & ancient domestication \\
\hline $\operatorname{Tr} 20$ & 53 & 0 & 29 & 0.403 & currently active \\
\hline $\operatorname{Tr} 21$ & & & & & currently active \\
\hline $\operatorname{Tr} 22$ & 0 & 0 & 0 & $d S=0$ & currently active \\
\hline $\operatorname{Tr} 23$ & & & & & currently active \\
\hline $\operatorname{Tr} 24$ & 57 & 6 & 23 & 0.28 & domestication? \\
\hline $\operatorname{Tr} 25$ & & & & & active? \\
\hline $\operatorname{Tr} 26$ & 15 & 0 & 3 & 0.058 & domestication? \\
\hline $\operatorname{Tr} 27$ & 55 & 0 & 19 & 0.118 & active? \\
\hline $\operatorname{Tr} 28$ & 9 & 0 & 6 & 0.629 & ancient domestication \\
\hline Tr29 & 27 & 0 & 12 & 0.312 & ancient domestication \\
\hline $\operatorname{Tr} 30$ & 5 & 0 & 2 & 0.328 & ancient domestication \\
\hline $\operatorname{Tr} 31$ & 21 & 3 & 11 & 0.359 & ancient domestication \\
\hline $\operatorname{Tr} 32$ & 7 & 0 & 6 & 1.444 & domestication? \\
\hline $\operatorname{Tr} 33$ & 30 & 0 & 8 & 0.14 & ancient domestication \\
\hline $\operatorname{Tr} 34$ & 23 & 0 & 9 & 0.192 & domestication? \\
\hline $\operatorname{Tr} 35$ & & & & & active? \\
\hline $\operatorname{Tr} 36$ & & & & & active? \\
\hline $\operatorname{Tr} 37$ & & & & & domestication? \\
\hline $\operatorname{Tr} 38$ & 12 & 0 & 5 & 0.196 & domestication? \\
\hline \multicolumn{6}{|c|}{ B. Differences between $X$. laevis and $X$. tropicalis, and presumptive status } \\
\hline LV1 & & & & & currently active \\
\hline LV2 & 466 & 237 & 202 & 0.285 & domestication? \\
\hline Lv3 & 472 & 63 & 210 & 0.194 & currently active \\
\hline Lv4 & 263 & 6 & 110 & 0.257 & ancient domestication \\
\hline
\end{tabular}


Table 4 Differences in transposase CDSs and amino acids, and presumptive status (Continued)

\begin{tabular}{|c|c|c|c|c|c|}
\hline Name & Nucleotide substitution & Nucleotide gap & Amino acid substitution & $\mathrm{dN} / \mathrm{dS}$ & Presumptive status \\
\hline Lv5 & & & & & domestication? \\
\hline Lv6 & & & & & currently active \\
\hline LV7 & 289 & 51 & 140 & 0.391 & ancient domestication \\
\hline LV8 & 293 & 57 & 141 & 0.301 & ancient domestication \\
\hline Lv9 & 303 & 42 & 154 & 0.341 & ancient domestication \\
\hline Lv10 & 302 & 36 & 161 & 0.463 & ancient domestication \\
\hline Lv11 & 335 & 42 & 172 & 0.358 & ancient domestication \\
\hline LV12 & 546 & 252 & 213 & 0.100 & domestication? \\
\hline LV13 & 359 & 198 & 164 & 0.365 & ancient domestication \\
\hline LV14 & 305 & 36 & 155 & 0.413 & ancient domestication \\
\hline Lv15 & 411 & 393 & 157 & 0.195 & domestication? \\
\hline Lv16 & 305 & 36 & 162 & 0.349 & ancient domestication \\
\hline Lv17 & 331 & 54 & 162 & 0.336 & ancient domestication \\
\hline LV18 & & & & & domestication? \\
\hline LV19 & 335 & 69 & 156 & 0.328 & ancient domestication \\
\hline LV20 & 323 & 66 & 165 & 0.497 & ancient domestication \\
\hline LV21 & 312 & 33 & 166 & 0.472 & ancient domestication \\
\hline LV22 & 356 & 72 & 193 & 0.521 & ancient domestication \\
\hline LV23 & 591 & 294 & 240 & 0.153 & active? \\
\hline LV24 & & & & & domestication? \\
\hline
\end{tabular}

(A) Differences between Nigerian and Asashima lines and (B) differences between $X$. laevis and $X$. tropicalis. $X$. tropicalis CDSs used in the analysis of differences with those of $X$. laevis are indicated by asterisks in Table 3B. The last column indicates the presumptive status of each $X K o /$ transposase

were not functional, because they were encoded by shorter CDSs that lacked certain lengths of conserved regions. For example, Tr36 lacked the C-terminal region, and $\operatorname{Tr} 34, \operatorname{Tr} 35, \operatorname{Tr} 37$, and $\operatorname{Tr} 38$ lacked the N-terminal regions conserved in the other XKol-Tpases. In contrast, most XKol-Tpases retained the DNA-binding motifs of Kolobok transposases, such as the $\mathrm{C} 2 \mathrm{CH}$ domain [36] and the THAP domain [37]. Therefore, at the very least, they partially retained their molecular functions.

Among the $62 \mathrm{XKol}$-Tpases, 24 (39\%) were grouped into seven triplet or doublet clusters of $X$. tropicalis and $X$. laevis XKol-Tpases on the molecular phylogenetic tree (subfamilies D1-D7). Their genes were located on homologous chromosome loci. All the dN/dS ratios between the $X$. tropicalis and $X$. laevis CDSs within each subfamily were less than 1.0. These results indicated that they were derived from an ancestral transposase gene domesticated before the divergence of the Хenopus genus. Among them, only five (21\%) of the XKol-Tpases (Tr10, Tr13, Lv4, Lv7, and Lv8 belonging to D1 or D5) retained all conserved Kolobok transposase motifs. The catalytic DDE motif was conserved only in these five proteins, whereas the other 19 lost this motif. In contrast, the $\mathrm{C} 2 \mathrm{CH}$ and PWP DNA-binding motifs were

Table 5 Full-length $X$. tropicalis XKol sequences

\begin{tabular}{|c|c|c|c|c|c|c|c|c|}
\hline Subfamily & CDS & CDS locus & Full-length $X \mathrm{kol}$ sequence & Length & TIR left & TIR right & TSD left & TSD right \\
\hline A1 & $\operatorname{Tr} 21$ & Chr04:107657217..107659511(+) & Chr04:107650836..107663091(+) & 12,216 & AGCGATTCTGACATGG & AGTGATACTGACAGTA & TTAA & TTAA \\
\hline $\mathrm{A} 1$ & $\operatorname{Tr} 22$ & Chr01:168455606.168457900(-) & Chr01:168451382..168460584(-) & 9163 & AGCGATTCTGACATGG & AGTGATACTGACAGTA & TTAA & TTAA \\
\hline $\mathrm{A} 1$ & $\operatorname{Tr} 23$ & Chr03:106168762..106171056(-) & Chr03:106165634..106173458(-) & 7785 & AGCGATTCTGACATGG & AGTGATACTGACAGTA & TTAA & TTAA \\
\hline A2 & $\operatorname{Tr} 8$ & Chr08:54548260.54550611(+) & Chr08:54546557..54553359(+) & 6763 & AGGAGAAGGAAAGGCT & AGGAAATGGCAAGCCA & TTAA & TTAA \\
\hline $\mathrm{A} 2$ & $\operatorname{Tr} 11$ & scaffold_609:18293..20635(-) & scaffold_609..15284:22344(-) & 7021 & AGGAGAAGGAAAGGCT & AGGAAATGGCAAGCCA & TTAA & ATAA \\
\hline A3 & $\operatorname{Tr} 14$ & scaffold_51:231432..233759(-) & scaffold_51..227047:240993(-) & 13,907 & AGGACATGTCAACCCC & AGGACGTGTCAACCCT & TТA & TTAA \\
\hline A3 & $\operatorname{Tr} 15$ & Chr01:83498100..83500424(+) & Chr01:83495893..83504753(+) & 8821 & AGGACATGTCAACCCC & AGGACGTGTCAACCAT & TTAA & TTAA \\
\hline A4 & $\operatorname{Tr} 25$ & Chr01:107512548..107514821(+) & Chr01:107510216..107521330(+) & 11,075 & AGGACAAGGAAAGCTT & AGGAAAATGAAAGTCA & TТA & ПTAA \\
\hline A5 & $\operatorname{Tr} 2$ & Chr02:100830975..100833458(+) & Chr02:100828018..100838339(+) & 10,282 & AGGGGAACTATCATGA & AGGGGATCTATCATGA & TTAA & TTAA \\
\hline A6 & $\operatorname{Tr} 9$ & Chr02:55486066..55488417(+) & Chr02:55483392..55492644(+) & 9213 & AGAGCAAGTAAAGTCG & AGAGCAAGGCAAGCTा & TTAA & TTAA \\
\hline
\end{tabular}

The position of full-length transposases, their left and right terminal sequences, and conservation of target sequences duplicated by the insertion of a transposon 
conserved in all 24 proteins. The $\mathrm{H} 2 \mathrm{CH}$ and AVPTIF domains, the latter of which was defined as conserved if each of its amino acids was substituted by similar ones, were highly conserved. Only four proteins in subfamily D6-2 or D7 (Tr4, Tr33, Lv9, and Lv13) lost the former domain, and two proteins in subfamily D7 ( $\mathrm{Tr} 4$ and Lv9) lost the latter domain. These results suggest that most of the domesticated XKol-Tpases retained their DNA-binding activity, but lost the catalytic activity necessary for transposition. These "transposases" may suppress transposons by competitively binding the target sequences of active transposases.

Fifteen other nonrepetitive XKol-Tpase genes were also found. Among them, only five proteins conserved the DDE motifs, whereas the $\mathrm{H} 2 \mathrm{CH}-, \mathrm{C} 2 \mathrm{CH}-$, PWF-, and AVPTIF motifs were conserved in 14, 11, 12, and 11 of the 15 proteins, respectively. Therefore, most of these XKol-Tpases may also have retained their DNA-binding activity but lost the ability to catalyze transposition. They may have been domesticated after the divergence of the two Xenopus species. Alternatively, they may constitute the remainder of ancestral domesticated transposase genes, and their orthologs may have been lost in other species. According to this study, 39 prospective XKol-Tpase genes (63\%) were apparently domesticated.

The best tblastn hits to XKol-Tpase in the GenBank database (excluding hits to the Xenopus sequence) were invertebrate sequences (data not shown). These included the cnidarian Acropora digitifera uncharacterized LOC107332277 mRNA (XM_015897006) and the Exaiptasia pallida uncharacterized LOC110237754 mRNA (XM_021043370). The lack of domesticated genes encoding Kolobok transposases orthologous to XKol-Tpases in other vertebrates suggests that domestication occurred after Xenopus diverged from other model animals, including mammals and birds. Molecular phylogeny suggested that independent domestication events occurred several times. If the XKol-Tpases located on nonhomologous loci had been domesticated independently, domestication may have occurred at least 16 times in Xenopus. This situation was the opposite of that reported for the Xenopus piggyBac superfamily $T x p B$ [9]. The $\operatorname{Txp} B$ family includes only one domesticated subfamily, Kobuta, and it was domesticated only once in the Xenopus ancestor.

\section{Currently active Kolobok transposases and the T2-MITE family}

Among the $62 \mathrm{XKol}$-Tpase genes, 23 (37\%) were repetitive, having interspersed multiple intact and/or truncated copies in the genome. Of these, 15 (65\%) retained all conserved motifs of the Kolobok superfamily transposase. We regard them as currently active transposases. The eight other repetitive XKol-Tpases had lost at least one conserved motif. We could not determine whether they were currently active.

Some "currently active" X. tropicalis XKol-Tpases were conserved between the Nigerian and Asashima lines. The CDSs of Tr6, Tr15, and Tr22 cloned from the Asashima line were nearly or exactly identical to those from the Nigerian line. Considering the distance between these two lines [35], this similarity may be explained by recent introgression or horizontal transfer of these genes from the original population of the Asashima line to that of the Nigerian line or vice versa. The CDSs of $\operatorname{Tr} 7, \operatorname{Tr} 8$, and $\operatorname{Tr} 20$ presented with 9,26 , and 53 nucleotide substitutions between the two lineages, respectively. However, their ORFs were conserved despite the large number of mutations. The "currently active" X. laevis XKol-Tpase Lv3 and the closely related $X$. tropicalis $\operatorname{Tr} 2$ had $472 \mathrm{nu}-$ cleotide substitutions and 63 nucleotide gaps, but their ORFs were also conserved (Table 4). This phenomenon may be explained by purifying selection because the $\mathrm{dN} /$ dS ratio between them was 0.194 . These results suggested that some $\mathrm{XKol}$ family transposons were currently active in the host and had also been domesticated by it. Domesticated XKol-Tpases belonging to the D1 and D5 subfamilies also conserved all Kolobok transposase motifs, including the catalytic DDE domain. Therefore, domestication and catalytic transposition activity may not be mutually exclusive in the XKol family. This apparent contradiction may be resolved by considering that the transposition catalysis of XKol-Tpases has been beneficial to the host.

Kolobok transposases probably transpose the T2-MITE family [38], which is predominant in Xenopus [13, 14, 16]. Certain subfamilies (T2-A1 and T2-C) may be "long-lived"; that is, they have retained transposition activity in the $X$. tropicalis lineage since before the divergence of $X$. laevis and $X$. tropicalis $[19,21]$. Our recent analysis of $X$. laevis revealed that some T2-MITE subfamilies have multiple identical copies, suggesting that they have recently been active (unpublished data). The long conservation activity of T2-MITE subfamilies in both species suggested that they may have contributed to host fitness through transposition. Recently, we reported that the T2-C subfamily tended to be located near the upstream regions of genes in $X$. tropicalis. The expression patterns of genes with upstream insertions were strongly correlated [22]. This distribution may indicate its function to be recruited by the host. For example, T2-C may include a cis-regulatory element. The insertion of a certain element into an upstream region may have been beneficial to the host; hence, it was conserved by natural selection. Recent studies have revealed that TE-derived sequences were recruited by hosts as cis-regulatory elements. TE transposition and amplification are important drivers of the evolution of the gene regulatory network [39]. The 
conservation of the Kolobok superfamily may be partially explained by pressure from the host to continue to activate the transposition of some T2-MITE subfamilies and to rewire their gene regulatory network.

\section{Conclusions}

Our results indicated that multiple massive XKol-Tpase domestication events occurred during Xenopus evolution and that XKol family transposons have complex, mutually beneficial relationships with their hosts. Long conservation of transposition activity and/or conserved catalytic and DNA-binding domains in certain XKol-Tpases suggest that they may benefit the host by catalyzing the transposition of long-lived T2-MITE subfamilies.

\section{Additional files}

\begin{abstract}
Additional file 1: Figure S1. Full-length multiple alignment of transposases predicted from prospective CDSs of XKol-Tpase genes with three outgroup transposases. Certain proteins predicted from the longest ORFs had excess $\mathrm{N}$-terminal amino acids compared with other proteins. We trimmed the corresponding $5^{\prime}$ regions from these prospective CDSs to align their start methionine codons with those in the others. This alignment was used in molecular phylogeny (Fig. 1). (PDF 50 kb)
\end{abstract}

Additional file 2: Figure S2. Repetitive XKol-Tpase CDSs (highlighted in red) were compared with their truncated copies by dot plot analyses (word size $=12$ ). Flanking upstream and downstream 1000 bp CDSs and truncated copy sequences are also included for comparison. (PDF $717 \mathrm{~kb}$ )

Additional file 3: Figure S3. XKol-Tpase CDSs and flanking upstream and downstream 2000 bp sequences of subfamily D1-D9 were compared by dot plot analyses (word size $=10)$. (PDF $319 \mathrm{~kb}$ )

Additional file 4: Figure S4. Multiple alignment of prospective transposase CDSs from the $X$. tropicalis and $X$. laevis genome databases and from CDSs cloned from $X$. tropicalis Nigerian and Asashima lineages. (PDF $167 \mathrm{~kb}$ )

Additional file 5: Table S1. Name for the Kolobok superfamily transposase query used in the tblastn search. Name for the source transposon in Repbase and protein length are shown. (XLSX $31 \mathrm{~kb}$ )

Additional file 6: Table S2. Primer sequences used in PCR to clone $X K O /$-Tpase CDSs from the $X$. tropicalis Nigerian and Asashima lineages. (XLSX $33 \mathrm{~kb}$ )

Additional file 7: Table S3. Results of blastn searches to identify truncated copies of XKol-Tpase genes. Prospective XKol-Tpase CDS used as a query, locus of the CDS, hit locus of the blastn search (e-value $<1 \mathrm{e}-100$ ), and its length (bp) are shown. (XLSX $30 \mathrm{~kb})$

\section{Acknowledgements}

We thank Dr. Akira Kawahara, Professor Emeritus of Hiroshima University, for his helpful suggestions and discussions. The two $X$. tropicalis lineages were kindly provided by the National Bio-Resource Project of the Ministry of Education, Culture, Sports, Science, and Technology (MEXT), Japan.

\section{Funding}

This study was supported by a Grant-in-Aid for Scientific Research from MEXT (No. 25440180).

\section{Availability of data and materials}

The datasets and materials generated and/or analyzed during the current study are available from the corresponding author upon reasonable request.

\section{Authors' contributions}

$\mathrm{AH}$ designed the analysis and experiment. AH performed the bioinformatics analysis. $\mathrm{AH}$ and SK performed the experiment. $\mathrm{AH}$ analyzed the results. $\mathrm{AH}$ wrote the manuscript. Both authors read and approved the final manuscript.

Ethics approval and consent to participate

Not applicable.

Consent for publication

Not applicable.

\section{Competing interests}

The authors declare that they have no competing interests.

\section{Publisher's Note}

Springer Nature remains neutral with regard to jurisdictional claims in published maps and institutional affiliations.

Received: 26 February 2018 Accepted: 6 June 2018

Published online: 16 June 2018

\section{References}

1. Finnegan DJ. Eukaryotic transposable elements and genome evolution. Trends Genet. 1989:5:103-7.

2. Wicker T, Sabot F, Hua-Van A, Bennetzen JL, Capy P, Chalhoub B, et al. A unified classification system for eukaryotic transposable elements. Nat Rev Genet. 2007:8:973-82.

3. Lohe AR, Moriyama EN, Lidholm DA, Hartl DL. Horizontal transmission, vertical inactivation, and stochastic loss of mariner-like transposable elements. Mol Biol Evol. 1995;12:62-72.

4. Brookfield JFY. The ecology of the genome - mobile DNA elements and their hosts. Nat Rev Genet. 2005;6:128-36.

5. Volff J-N. Turning junk into gold: domestication of transposable elements and the creation of new genes in eukaryotes. Bioessays. 2006;28:913-22.

6. Jangam D, Feschotte C, Betrán E. Transposable element domestication as an adaptation to evolutionary conflicts. Trends Genet. 2017;33:817-31.

7. Sinzelle L, Izsvák Z, Ivics Z. Molecular domestication of transposable elements: from detrimental parasites to useful host genes. Cell Mol Life Sci. 2009;66:1073-93.

8. Alzohairy AM, Gyulai G, Jansen RK, Bahieldin A. Transposable elements domesticated and neofunctionalized by eukaryotic genomes. Plasmid. 2013;69:1-15.

9. Hikosaka A, Kobayashi T, Saito Y, Kawahara A. Evolution of the Xenopus piggyBac transposon family TxpB: domesticated and untamed strategies of transposon subfamilies. Mol Biol Evol. 2007:24:2648-56.

10. Feschotte C, Zhang X, Wessler SR. Miniature inverted-repeat transposable elements and their relationships to established DNA transposons. In: Craig NL, et al., editors. Mobile DNA II. Washington: ASM Press; 2002. p. 1093-110.

11. El Amrani A, Marie L, Aïnouche A, Nicolas J, Couée I. Genome-wide distribution and potential regulatory functions of AtATE, a novel family of miniature inverted-repeat transposable elements in Arabidopsis thaliana. Mol Gen Genomics. 2002;267:459-71.

12. Avramova Z, Tikhonov A, Chen M, Bennetzen JL. Matrix attachment regions and structural colinearity in the genomes of two grass species. Nucleic Acids Res. 1998;26:761-7.

13. Hikosaka A, Yokouchi E, Kawahara A. Extensive amplification and transposition of a novel repetitive element, Xstir, together with its terminal inverted repeat in the evolution of Xenopus. J Mol Evol. 2000:51:554-64.

14. Hikosaka A, Kawahara A. Lineage-specific tandem repeats riding on a transposable element of MITE in Xenopus evolution: a new mechanism for creating simple sequence repeats. J Mol Evol. 2004;59:738-46.

15. Ugarkovic D. Functional elements residing within satellite DNAs. EMBO Rep. 2005;6:1035-9.

16. Ünsal K, Morgan GT. A novel group of families of short interspersed repetitive elements (SINEs) in Xenopus - evidence of a specific target site for DNA-mediated transposition of inverted-repeat SINEs. J Mol Biol. 1995;248: 812-23.

17. Izsvák Z, Ivics Z, Shimoda N, Mohn D, Okamoto H, Hackett PB. Short inverted-repeat transposable elements in teleost fish and implications for a mechanism of their amplification. J Mol Evol. 1999;48:13-21. 
18. Kapitonov W, Jurka J. Kolobok, a novel superfamily of eukaryotic DNA transposons. Repbase Reports. 2007;7:111-22.

19. Hikosaka A, Kawahara A. A systematic search and classification of T2 family miniature inverted-repeat transposable elements (MITEs) in Xenopus tropicalis suggests the existence of recently active MITE subfamilies. Mol Gen Genomics. 2010;283:49-62.

20. Session AM, Uno Y, Kwon T, Chapman JA, Toyoda A, Takahashi S, et al. Genome evolution in the allotetraploid frog Xenopus laevis. Nature. 2016; 538:336-43.

21. Hikosaka A, Nishimura K, Hikosaka-Katayama T, Kawahara A. Recent transposition activity of Xenopus T2 family miniature inverted-repeat transposable elements. Mol Gen Genomics. 2011;285:219-24.

22. Hikosaka A, Uno Y, Matsuda Y. Distribution of the T2-MITE family transposons in the Xenopus (Silurana) tropicalis genome. Cytogenet Genome Res. 2015:145:1-13.

23. Xenopus laevis genome (J-Strain 9.2). ftp://ftp.xenbase.org/pub/Genomics/ JGI/Xenla9.2/. 2017. Accessed 29 Aug 2017.

24. Xenopus tropicalis genome (Nigerian 9.1). ftp://ftp.xenbase.org/pub/ Genomics/JGI/Xentr9.1/. 2017. Accessed 29 Aug 2017.

25. Bao W, Kojima KK, Kohany O. Repbase update, a database of repetitive elements in eukaryotic genomes. Mob DNA. 2015;6:11.

26. RepBase Release 22.09. http://www.girinst.org/server/archive/RepBase22.09/. 2017. Accessed 27 Sep 2017.

27. Altschul SF, Gish W, Miller W, Myers EW, Lipman DJ. Basic local alignment search tool. J Mol Biol. 1990;215:403-10.

28. Kumar S, Stecher G, Tamura K. MEGA7: molecular evolutionary genetics analysis version 7.0 for bigger datasets. Mol Biol Evol. 2016;33:1870-4.

29. Edgar RC. MUSCLE: multiple sequence alignment with high accuracy and high throughput. Nucleic Acids Res. 2004;32:1792-7.

30. Saitou N, Nei M. The neighbor-joining method: a new method for reconstructing phylogenetic trees. Mol Biol Evol. 1987;4:406-25.

31. Jones DT, Taylor WR, Thornton JM. The rapid generation of mutation data matrices from protein sequences. Bioinformatics. 1992;8:275-82.

32. Rice P, Longden I, Bleasby A. EMBOSS: the European molecular biology open software suite. Trends Genet. 2000;16:276-7.

33. Yang Z, Nielsen R. Estimating synonymous and nonsynonymous substitution rates under realistic evolutionary models. Mol Biol Evol. 2000;17:32-43.

34. Yang Z. PAML 4: phylogenetic analysis by maximum likelihood. Mol Biol Evol. 2007:24:1586-91.

35. Kashiwagi K, Kashiwagi A, Kurabayashi A, Hanada H, Nakajima K, Okada M, et al. Xenopus tropicalis: an ideal experimental animal in amphibia. Exp Anim. 2010:59:395-405.

36. Meng Q, Chen K, Ma L, Hu S, Yu J. A systematic identification of Kolobok superfamily transposons in Trichomonas vaginalis and sequence analysis on related transposases. J Genet Genomics. 2011;38:63-70.

37. Roussigne M, Kossida S, Lavigne AC, Clouaire T, Ecochard V, Glories A, et al. The THAP domain: a novel protein motif with similarity to the DNA-binding domain of $P$ element transposase. Trends Biochem Sci. 2003:28:66-9.

38. Hellsten U, Harland RM, Gilchrist MJ, Hendrix D, Jurka J, Kapitonov V, et al. The genome of the western clawed frog Xenopus tropicalis. Science. 2010; 328:633-6.

39. Chuong EB, Elde NC, Feschotte C. Regulatory activities of transposable elements: from conflicts to benefits. Nat Rev Genet. 2016;18:71-86.

\section{Ready to submit your research? Choose BMC and benefit from:}

- fast, convenient online submission

- thorough peer review by experienced researchers in your field

- rapid publication on acceptance

- support for research data, including large and complex data types

- gold Open Access which fosters wider collaboration and increased citations

- maximum visibility for your research: over $100 \mathrm{M}$ website views per year

At BMC, research is always in progress.

Learn more biomedcentral.com/submissions 